Voix et Images

\title{
La vie comme un roman ou la stylisation du réel
}

\section{Frances Fortier}

Volume 32, numéro 2 (95), hiver 2007

Féminin/Masculin. Jeux et transformations

URI : https://id.erudit.org/iderudit/016316ar

DOI : https://doi.org/10.7202/016316ar

Aller au sommaire du numéro

\section{Éditeur(s)}

Université du Québec à Montréal

\section{ISSN}

0318-9201 (imprimé)

1705-933X (numérique)

Découvrir la revue

Citer ce compte rendu

Fortier, F. (2007). Compte rendu de [La vie comme un roman ou la stylisation du réel]. Voix et Images, 32(2), 129-134. https://doi.org/10.7202/016316ar d'utilisation que vous pouvez consulter en ligne.

https://apropos.erudit.org/fr/usagers/politique-dutilisation/ 
R O M A N

La vie comme un roman ou la stylisation du réel

$++$

FRANCES FORTIER

Université du Québec à Rimouski

Dire le réel à même une forme romanesque exige pour le moins une bonne dose d'ingéniosité formelle pour en pervertir les codes, un brin d'effervescence imaginaire pour rêver la vie et une écriture pour transmuter l'expérience en littérature. Sous couvert de fictions familiales qui racontent le passage à l'âge adulte d'une protagoniste enfant évoluant dans un univers marqué, à des degrés divers, par la violence psychologique, sociale ou physique, les textes retenus ici revendiquent éloquemment leur inscription dans l'espace littéraire tant par les défis formels qu'ils relèvent que par la présence, en filigrane, de modèles réinterprétés. Trois réalités, éloignées culturellement et géographiquement, se voient ainsi stylisées par le recours à un dispositif qui autorise toutes les audaces: le Québec d'avant les années 1970 évoqué par le biais d'une autobiographie à la troisième personne qui montre la prégnance de la littérature dans l'apprentissage de la vie; la Géorgie post-soviétique où la tonalité ironique laisse entrevoir une allégorie politique au gré des mariages constamment reportés d'une jeune fille de seize ans; le Montréal multiculturel contemporain où le naufrage identitaire d'une jeune femme meurtrie par son enfance passe par des réduplications qui hypothèquent les dérives autofictionnelles. Ces postures, loin de trahir la réalité, donnent l'impression de s'en approcher au plus près.

À la manière d'une autobiographie déguisée, l'émouvant récit de Madeleine Ouellette-Michalska, L'apprentissage ${ }^{1}$, met en scène une protagoniste féminine anonyme, montrée à diverses étapes de sa vie, de l'enfance rêveuse à l'âge adulte qui la fait écrivaine: «Elle a fini par rejoindre son rêve. Plus tard et maintenant sont devenus synonymes. La vie commence à prendre des allures de fiction.» (69) Un tel énoncé, emblématique des stratégies formelles qui permettent de contourner les poncifs de la quête identitaire, déjoue tous les pièges de l'écriture de soi, qu'il s'agisse de l'idéalisation du destin, de l'héroïsation du sujet ou de l'illusion rétrospective, en même temps qu'il marque, paradoxalement, la singularité du parcours évoqué. Cette « elle» sans patronyme, désignée par une série de vocables relevant de

$$
++
$$

1 Madeleine Ouellette-Michalska, L'apprentissage, Montréal, XYZ éditeur, coll. «Romanichels», 2006, 134 p. 
la chronologie - l'enfant, l'adolescente, l'enseignante, l'adulte, la femme - et qui évolue parmi une théorie de personnages familiers - le père, la mère, les huit sœurs, les cousins, les tantes - ou plus exotiques - les touristes, la parenté américaine, les bohémiens, le violoniste ambulant, la danseuse - tout aussi indéterminés, apprend la vie au gré d'expériences qui n'ont rien d'exceptionnel. Le découpage du texte en six chapitres autorise une progression linéaire qui va mener la fillette lucide mais docile au monde strictement quadrillé entre le proche et le lointain, l'ici et l'ailleurs, l'accessible et l'interdit, jusqu'à l'âge d'écrire, où

[e]lle dira le temps de l'amour, les nuits folles, le désir d'étreindre. Elle dira l'enfantement, les mutations et les replis, la passion qui empêche le jour de s'éteindre et le monde de mourir. [...] Elle écrira aussi le tumulte de certains soirs, l'effort et la lassitude, les mots vengeurs, le désespoir. Dans divers épisodes, elle racontera des bribes de vie ayant appartenu à des générations différentes, faisant ressortir leur commune appartenance à des projets, à des élans, à des douleurs similaires. Elle dessinera le contour de visages formés de traits joints par des morceaux de temps distincts, sachant que la vie, soumise à la loi de la dégénérescence, parviendra à les unifier. Et dans chacune de ces ébauches, elle dira l'inutile distance que les gens placent entre eux, tous se trouvant un jour ou l'autre réunis dans une même mort et un même oubli. (133)

À l'individualité d'un sujet qui n'aurait de cesse de singulariser son existence, le texte préfère un sujet anonyme, pétri d'expériences communes, le langage, le territoire, la mort, le désir, l'amour, et qui parvient néanmoins à «être soi» (118).

Une stylisation extrêmement raffinée soutient cette histoire des «choses habituelles de la vie» (27) qui s'esquisse en petites touches subtiles, en allusions, en scènes de genre saisies en instantané. On comprend, sans que cela ne soit toujours expressément précisé, que l'enfance du personnage s'est déroulée au moment de la Seconde Guerre mondiale, dans un domaine familial non loin de Notre-Damedu-Portage, dans une "maison de la falaise» sur la route de Gaspé; on ressent l'agression du cousin R. qui, haletant, "fait sauter le foulard de velours, l'agrafe du soutien-gorge, et masse les seins rudement» (86) comme on éprouve le malaise du silence autour de la table familiale, alors que la parole sert «moins de relais que de voie d'évitement» (96). L'effet est encore renforcé par la temporalité, alors que le récit, entièrement écrit au présent, permet une adhésion franche aux faits racontés, sans filtre médiateur, et crée un climat d'authenticité à l'occasion raffermi par les projections et les annonces. Ce présent tendu vers l'avenir incarne littéralement le propos, où «la vie sait prendre soin de la vie» (115) mieux que tous les scénarios préalablement imaginés: "La vie est un rêve d'enfant qui ne se termine jamais.» (132)

Mais L'apprentissage est d'abord et avant tout une pétition d'écrivain, et la singularité de l'expérience réside dans le rapport à la littérature, qui a présidé à toutes les découvertes, permis à l'occasion de comprendre les situations - «Elle est passée d'un monde balzacien où tout était rangé, pesé, à un monde tolstoïen régi par 
le désordre et la spontanéité» (111) - ou de lier la réalité au rêve, comme dans cette première maison «qui contenait tout ce dont rêve la littérature: des objets rares, des armoires et des coffres regorgeant de livres, de linge, de réserves, une atmosphère chargée de suffisamment de tensions et d'évocations pour enflammer l'imaginaire » (78). Au terme du récit, «la femme qui écrit» apparaîtra lestée de tous ses apprentissages passés, et bien déterminée à vivre cette passion du langage qui l'aura fait passer d'un âge où «l'art est une copie du réel, un calque des choses observées » (34) à celui de la maturité où il s'agit «maintenant de sortir de la littérature, trouver l'écho des voix, la résonance des mots. Elle veut traduire la passion d'être, l'ultime parole pouvant toucher le noyau irradiant et silencieux de toute vie» (134).

Dans un tout autre registre, le troisième roman d'Elena Botchorichvili, intitulé Faïna ${ }^{2}$, propose une galerie de personnages truculents évoluant dans une Géorgie d'avant Mikhail Gorbachev autour d'une lignée matriarcale composée de Noutsa Tsereteli, veuve à seize ans d'un prince géorgien fusillé et qui ne remarchera jamais, de sa sœur Nadia qui reçoit chaque été tous les enfants de la famille dans sa maison de Koutaïssi, de sa fille Oliko, mariée à seize ans à un médecin unijambiste qui pratique des avortements clandestins et de sa petite-fille Fafotchka, la Faïna du titre, qui à seize ans s'apprête à épouser un jeune homme aux sourcils noirs, sans savoir «bien sûr, qu'elle allait se marier presque tous les ans» (28). Loufoque, le récit est ponctué de funérailles théâtrales, de "festins géorgiens » (84) gargantuesques, d'un fauteuil de velours qu' on roule à table "comme on roule un canon" (20), de contrebande d'aiguilles à coudre, de héros militaires qui n'ont jamais connu le feu, de soulier à talon qu'on remplit de vin pour porter un toast aux mères.

Sous un comique de dérision, cette histoire de femmes s'écrit sur une trame de fond politique et laisse affleurer le tragique de la vie en régime totalitaire ${ }^{3}$. Géorgienne d'origine, l'auteure, qui vit présentement à Montréal, excelle à rendre concrets les effets des réorientations successives mises de l'avant par Joseph Staline, Nikita Khrouchtchev, Léonide Brejnev et autres consorts, montrant que «[l]es dirigeants changeaient, mais pas la vie» (85) à Tbilissi :

Le mariage de Fafotchka, celui du moment, a encore dû être reporté, et on a vendu l'appartement avec vue sur le monument au Révolutionnaire. D'ailleurs, la statue avait été depuis longtemps déboulonnée; il ne restait plus que le socle. Mais même le socle, les gens avaient continué de le souiller de saletés et de peinture rouge sang. Fafotchka et Oliko, toujours en guerre, sont revenues aux Sololaki, où étaient réinstallée Noutsa, remariée, presque aveugle. On a vendu les verres grands et petits, les vases de cristal, même la robe de mariée de Fafotchka - elle ne pouvait plus l'enfiler - et on a mangé tout ce que la vente a rapporté. (88)

Une vingtaine de tableautins picaresques - où perce la roublardise nécessaire à la survie, pour contrer les menaces de délations et autres vicissitudes liées à la vie

2 Elena Botchorichvili, Faïna, traduit du russe par Carole Noël, Montréal, Boréal, 2006, 108 p. 3 L'exubérance du ton rappelle Les noces dans la maison de Bohumil Hrabal (Paris, Éditions du Seuil, coll. « Points», 1990 [1986]). 
sous l'empire soviétique - reconduisent en filigrane, avec une ironie fine, tous les clichés, des appartements bondés à la culture du secret, des beuveries quotidiennes aux tractations du marché noir, des employés qui «fumaient dans les coins en se plaignant qu'il était impossible de vivre avec leur salaire» (73) aux médecins qui ne pratiquaient plus puisque, «au beau milieu d'une opération, l'électricité manquait ou on coupait l'eau» (87), sans compter le "camarade haut placé» (33) qui aplanit à l'occasion les difficultés. Ce récit à double entente, qui dissimule les horreurs sous une loufoquerie de surface, reprend à son compte les mécanismes de dissimulation propres au langage, évoqués en leitmotiv tout au long du texte: «Les mots, c'est quoi? Du vent!» $(18,38,51)$ et s'apparente au journal de Volodia le médecin, ce journal qui demeure incompréhensible aux yeux de sa descendance (106) car il métaphorise les situations. Et le texte ainsi d'associer le féminin à l'essentiel de la vie - une féminité marquée par la beauté et le volume des seins, constamment réitéré et les aléas de la politique aux régimes alimentaires: l'énorme seau de caviar noir d'avant Lénine (9), le beurre salé de Brejnev, les amourettes de Franklin Roosevelt (61) après la guerre, le maïs de Khrouchtchev «quand tout le pays cultivait le maïs, mangeait le maiis et appelait $\mathrm{K}$. - celui qui entassait le peuple dans des cuisines aux plafonds bas comme des bunkers - "Maïstchik" » (77) et "les cuisses de Bush » qui viendraient plus tard (81). L'histoire s'arrête avec la glasnost et la perestroika de Gorbatchev en une sorte de fable où l'idylle amoureuse apparaît comme le remède à toutes les déceptions du politique.

Outre sa tonalité ironique, le récit séduit encore par la vivacité du rythme narratif, chaque scène ne dépassant pas quatre pages, et une structuration par reprises de motifs, tel ce piano à queue qui revient constamment dans l'histoire, d'abord abandonné sur un quai entre Berlin et Tbilissi, puis thêâtre d'un meurtre sans motif et qui a abouti dans la maison de Nadia où il est resté muet pendant des décennies avant d'être racheté par un assistant dentaire allemand: «Et ce piano à queue, sur lequel le taciturne Andro Kobaïdze avait été déposé, couvert de fleurs comme de médailles, sur lequel avaient dansé les enfants, sur lequel une fois Fafotchka s'était vautrée toute nue, les jambes ouvertes, ce piano à queue est reparti pour Berlin. » (90) La narration procède ainsi par cumul, exploitant toutes les facettes de l'allégorie en reprenant systématiquement un même jeu d'objets, d'actes et de lieux qui gagnent en épaisseur au fil du récit.

À l'inverse, dans Alia de Mélikah Abdelmoumen ${ }^{4}$, le tragique du propos, comme sa cause, sont donnés d'entrée de jeu. La narratrice, nommée Alia Ben Fasser, jeune femme dans la trentaine née d'un couple violent, apprend dès le premier tableau l'existence d'un frère jumeau. Alia, écrivaine, a publié deux tomes autobiographiques qui lui ont valu un procès intenté par ses parents et une célébrité médiatique; ce frère, Henri M. Dolbeic, artiste performeur parisien à la carrière internationale, finira par s'immoler par le feu «devant une grande fresque faite de débris de miroirs, d'éclats de verre multicolores dont le dessin formera les mots:

$$
++
$$

4 Mélikah Abdelmoumen, Alia, Montréal, Marchand de feuilles, 2006, 186 p. 
"pour Alia" » (167). Le récit trouve sa résolution dans l'apaisement d'un amour enfin accepté, celui d'un Blaise Robert inopinément surgi dans sa vie.

Dédié entre autres à Serge Doubrovsky avec une citation de Bret Easton Ellis tirée de Lunar Park ${ }^{5}$ en épigraphe, ce roman apparaît vite comme un exercice de déconstruction des pratiques de l'autofiction, alors que les écrits autofictionnels de la narratrice intitulés Autofragmentations sont le moteur de la narration, à la fois sources de ses ennuis, prétextes à un questionnement irrésolu sur la porosité de la frontière entre le vrai et l'imaginé, monuments érigés à son narcissisme, cris de douleur exacerbés, désirs de vengeance, en une mise à plat systématique des enjeux reconnus de ce genre de textes. Ponctué de trois segments intitulés "Autoadmonestation» numérotés, le récit au je met en scène diverses «Alias» (173), en de multiples postures affectives, de l'enfant maltraitée d'Ali baba à la jeune femme aux comportements autodestructeurs, en un curieux mélange d'esthétique trash et d'envolées fleur bleue. Le récit d'un cauchemar vient en bout de course redoubler le propos tout en lui ménageant une issue heureuse marquée par le dernier mot « Appelezmoi moi» (177):

Cette fois, nous sommes plusieurs dans la cellule. Il y a, jouant dans son coin avec une poupée rabougrie et deux ou trois crayons à colorier, Alia, cinq ans, reconnue coupable d'avoir feint une angine pour attirer l'attention de ses parents. Veillant sur elle, retenant mal ses larmes, se trouve Alia, neuf ans, condamnée à perpétuité pour avoir rêvé la nuit qu'un méchant assassinait ses parents. À côté, Alia, quatorze ans, coupable d'avoir déjà couché avec un garçon qui ne lui plaisait pas, sans y réfléchir, sans y prendre aucun plaisir et sans moyen de contraception. Et puis il y a Alia, vingt-deux ans, qui pleure aussi dans son coin, condamnée quant à elle pour alcoolisme et pour avoir adopté tous les autres types de comportements autodestructeurs, sur une période prolongée.

Enfin, assise sur le petit tabouret qui est toujours là, au même endroit, dans tous mes cauchemars carcéraux, il y a moi, Alia Ben Fasser, trente-et-quelques années, coupable d'avoir toujours dit qu'elle n'a rien fait. (174)

Cette fragmentation identitaire s'exprime encore par la matérialité même du texte, qui rapatrie sous trois parties encadrées par un Prologue (11) et un Post-Scriptum (181) - I. Le côté de Dolbeic (15) II. The Man I Love (57) III. Le côté des Ben Fasser (135) - au-delà d'une trentaine de textes brefs numérotés, qui se réclament d'une sorte d'intermédialité, convoquant tour à tour l'écriture, le sous-titrage, les standards de jazz, une série policière télévisée, les performances de l'art contemporain, une chanson de Charles Aznavour, qui non seulement servent le propos mais engendrent littéralement l'écriture. Alia fait ainsi figure d'un exercice savant, nourri de préceptes théoriques mis à l'épreuve d'une fragilité émotive qui sait démonter les miroirs factices.

$$
++
$$

5 Bret Easton Ellis, Lunar Park, New York, Knopf, 2005. 
Au-delà de la singularité de leur propos et de leur facture, au-delà de la diversité identitaire, culturelle ou générationnelle de leurs protagonistes, leur étonnant dénouement heureux rapproche ces trois textes: "la femme qui écrit» de Madeleine Ouellette-Michalska est au seuil d'une vie nouvelle, la Faïna de Botchorichvili découvre l'amour à sa descente de l'avion qui la ramène de Moscou et la Alia d'Abdelmoumen vit sereinement depuis longtemps avec son compagnon. Comme si ces bildungroman contemporains, réinterprétés au féminin, entendaient décrire des trajectoires individuelles où les contraintes, paradoxalement, disent la force de la résilience et le pouvoir du bonheur. Pourquoi pas? 